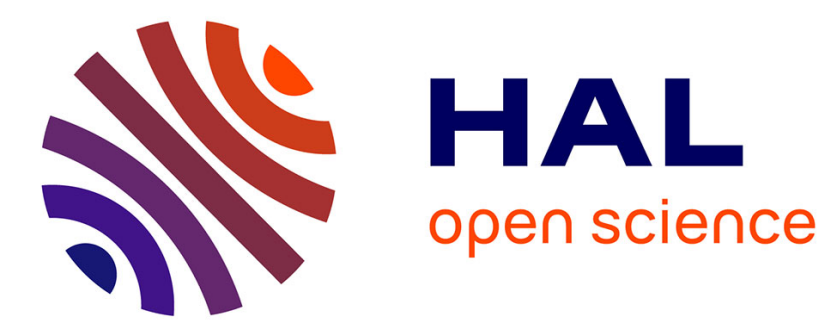

\title{
Collisional dissipation of the laser-induced alignment of ethane gas: A requantized classical model
}

\author{
J.-M. Hartmann, C. Boulet, H. Zhang, F. Billard, O. Faucher, B. Lavorel
}

\section{To cite this version:}

J.-M. Hartmann, C. Boulet, H. Zhang, F. Billard, O. Faucher, et al.. Collisional dissipation of the laser-induced alignment of ethane gas: A requantized classical model. Journal of Chemical Physics, 2018, 149 (15), pp.154301. 10.1063/1.5046899 . hal-01944596

\section{HAL Id: hal-01944596 \\ https://hal.sorbonne-universite.fr/hal-01944596}

Submitted on 4 Dec 2018

HAL is a multi-disciplinary open access archive for the deposit and dissemination of scientific research documents, whether they are published or not. The documents may come from teaching and research institutions in France or abroad, or from public or private research centers.
L'archive ouverte pluridisciplinaire $\mathbf{H A L}$, est destinée au dépôt et à la diffusion de documents scientifiques de niveau recherche, publiés ou non, émanant des établissements d'enseignement et de recherche français ou étrangers, des laboratoires publics ou privés. 


\title{
Collisional dissipation of the laser-induced alignment of ethane gas.
}

\section{A requantized classical model}

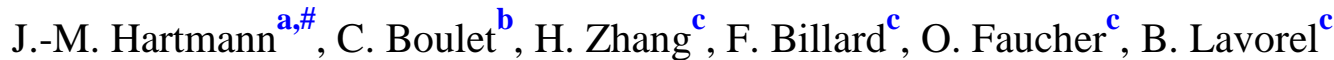 \\ a Laboratoire de Météorologie Dynamique/IPSL, CNRS, École polytechnique, Sorbonne \\ Université, École normale supérieure, PSL Research University, F-91120 Palaiseau, France. \\ \#Corresponding author: jean-michel.hartmann@1md.polytechnique.fr \\ ${ }^{\mathbf{b}}$ Institut des Sciences Moléculaires d'Orsay, CNRS, Université Paris-Sud, Université Paris- \\ Saclay, Orsay F-91405, France. \\ ${ }^{c}$ Laboratoire Interdisciplinaire Carnot de Bourgogne (ICB), UMR 6303 CNRS-Université, \\ Bourgogne-Franche Comté, 9 Ave. A. Savary, BP 47 870, F-21078 Dijon Cedex, France.
}

\begin{abstract}
We present the first theoretical study of the collisional dissipation of the alignment of a symmetric-top molecule (ethane gas) impulsively induced by a linearly polarized nonresonant laser field. For this, Classical Molecular Dynamics Simulations (CMDS) are carried out for an ensemble of $\mathrm{C}_{2} \mathrm{H}_{6}$ molecules based on knowledge of the laser-pulse characteristics and on an input intermolecular potential. These provide, for a given gas pressure and initial temperature, the orientations of all molecules at all times from which the alignment factor is directly obtained. Comparisons with measurements show that these CMDS well predict the permanent alignment induced by the laser pulse and its decay with time but, as expected, fail in generating alignment revivals. However, it is shown that introducing a simple requantization procedure in the CMDS "creates" these revivals and that their predicted dissipation decay agrees very well with measured values. The calculations also confirm that, as for linear molecules, the permanent alignment of ethane decays more slowly than the transient revivals. The influence of the intermolecular potential is studied as well as that of the degree of freedom associated with the molecular rotation around the symmetry axis. This reveals that ethane practically behaves as a linear molecule because the intermolecular potential is only weakly sensitive to rotation around the C-C axis.
\end{abstract}




\section{INTRODUCTION}

Aligning molecules with intense laser pulses is a well-established technique ${ }^{1,2}$. The alignment-dependent potential felt by anisotropic polarizable molecules immersed in a strong laser field, confines the most polarizable axis along the field polarization direction. When the pulse duration is much shorter than the rotational period of the molecule, the laser-molecule interaction is non-adiabatic and molecules exhibit periodic revivals of alignment after the pulse $^{3,4}$. This so-called field-free molecular alignment is highly desirable in a large variety of fields such as molecular tunnel-ionization 5,6 and orbital imaging 7 , high-order harmonic generation $^{8}$, attosecond electronics ${ }^{9}$, and molecule solvation dynamics ${ }^{10}$. Various approaches have been used to measure the field-free molecular alignment, mainly categorized as either ionization-fragmentation imaging techniques ${ }^{11,12}$ or nonlinear pure optical techniques ${ }^{13,14}$. The latter are particularly well suited when the alignment decreases quickly due to intermolecular collisions, as in high-pressure gases ${ }^{15-17}$, or to molecule-solution interactions, as in He nano-droplets 10,18 . The excitation of molecules with a non-resonant short pump pulse can be described through a cascaded rotational stimulated Raman effect that creates a rotational wavepacket and leads to energy deposition in the rotational motion. This energy then relaxes to the translational degrees of freedom through collisions, a process that was tracked in the time domain ${ }^{19,20}$. The concomitant elevation of temperature and pressure inside the sample produces a thermal expansion and an acoustic-wave which have been detected photo-acoustically ${ }^{21}$. Note that the exchange of energy between the pump electric field and the molecules can also be measured through dichroism ${ }^{22}$.

Field-free alignment, as a time-resolved technique, can provide a means to investigate collisional effects in molecular samples. An example is the determination of collisional linewidths from the recording of the time-dependent birefringence at moderate pressures ${ }^{23}$. It is thus an alternative to frequency-domain Raman spectroscopy. Moreover, as pointed out in the pioneering studies of Refs. 24,25, it could be possible to disentangle the effects of rotational population relaxation and pure phase decoherence by using the dissipation of field free alignment. This ability relies on the fact that the field-free molecular alignment exhibits two different contributions known as the transient and permanent alignments, which have different sensitivities to the various collisional processes. As discussed in Ref. 16, the permanent alignment corresponds to a population effect whereas the transient alignment reflects the coherence within the rotational excited wavepacket. While all collisions inducing decoherence $(J$-changing inelastic collisions, $J$-preserving elastic reorienting collisions, $J$ - and 
$M$-preserving pure dephasing elastic collisions) reduce the transient alignment, the decrease of the permanent component only results from those which change the orientation of the rotational angular momentum. It is thus possible, in principle, to discriminate different contributions to the dissipation of alignment by simultaneously measuring the decays of permanent and transient alignments under the same gas conditions. The first work in this direction, made in pure $\mathrm{CO}_{2}$ gas and $\mathrm{CO}_{2}$ in mixtures with rare gases at low pressure ${ }^{15}$, was then followed by a study for nitrogen molecules ${ }^{26}$. The analysis of the data demonstrated that inelastic collisions dominate the decoherence in the gas phase. More interesting, the decay of both components of field-free molecular alignment was then measured in OCS excited by a strong terahertz single cycle pulse ${ }^{27}$, which showed that the permanent alignment decays 3 times slower that the transient one. The explanation for this difference was given in Ref. 28 , for pure $\mathrm{CO}_{2}$ gas, by using two independent models for the collisional relaxation of molecular alignment: A quantum one, based on the $M$-dependent Energy Corrected Sudden (ECS) model, and a nearly classical one, using requantized Classical Molecular Dynamics Simulations (rCMDS). The main feature exhibited by both approaches, which lead to results in excellent agreement, is a propensity of collisions to conserve the orientation of the molecular angular momentum. Consequently, the permanent alignment decays slower than the transient one. This was later on confirmed by the very good agreement between the computed and measured decays of the permanent and transient alignments in high-pressure pure $\mathrm{CO}_{2}$ gas as well as $\mathrm{CO}_{2}-\mathrm{He}$ and $\mathrm{CO}_{2}$-Ar mixtures ${ }^{16,17,29}$. However, all the above mentioned experimental and theoretical studies of the collisional dissipation of the laserinduced alignment were made for linear molecules only, despite the obvious interest of studying the influence of the molecular symmetry. It is only very recently that dissipation for a nonlinear species (the symmetric-top molecule ethane $\mathrm{C}_{2} \mathrm{H}_{6}$ ) has been investigated experimentally ${ }^{30}$. Again, the permanent alignment shows a slower decay compared to that of the transient alignment, indicating the potentially general applicability of the propensity rule to nonlinear polyatomic molecules. However, while quantum calculations of the alignment of symmetric tops under collision-free conditions have been made (eg Ref. 30 and those there in), no theoretical approach and thus no simulations have been proposed so far for (gas pressure) conditions making dissipation processes significant.. This lack of models and computations of the collisional dissipation of the alignment of symmetric-top molecules is the justification of this paper. The latter presents an approach based on rCMDS for symmetric-top 
species (Sec. II) which is applied to ethane gas with (Sec. III) comparisons with experimental results ${ }^{30}$, and a study of the influence of the symmetric-top geometry.

\section{COMPUTATIONAL PROCEDURE AND DATA USED}

\section{A. Classical Molecular Dynamics Simulations (CMDS)}

CMDS were performed for an ensemble of $N$ ethane molecules at pressure $P$ and initial temperature $T$ as follows. First the molecules' orientations and center-of-mass positions [inside a cubic box of side length $\left(N k_{\mathrm{B}} T / P\right)^{1 / 3}$ ] were chosen randomly with the constraint that molecules should be at least 7 A away from each other (in order to avoid unphysical situations associated with too close pairs). Then the rotational angular momentum and center of mass velocity vectors were defined with random orientations and modulus verifying the Boltzmann statistics. These molecular parameters were then propagated in time using periodic boundary conditions, a closest neighbors sphere and the quaternion approach, as described in Ref. 31. The effects of the laser pulse and of intermolecular collisions appear in the equations through the subsequent force and torque applied to each molecule. The torque on molecule $m$ due to the laser is the vector product of the electric field $\vec{E}(t)$ and induced dipole $\vec{\mu}_{m}(t)$. The

latter is given by $\vec{\mu}_{m}(t)=\overrightarrow{\vec{\alpha}}_{m}(t) \cdot \vec{E}(t)$, where the polarizability tensor $\overrightarrow{\vec{\alpha}}_{m}(t)$ in the laboratory frame is computed from knowledge of its value in the molecular frame and of the orientation of the molecule (i.e. of the quaternions). For the force and torque resulting from intermolecular collisions, they are easily obtained from the positions and orientations of the interacting molecules and a parameterization of the site-site forces 32.33 (see below). Using the input data described there after, computations were made for ethane gas initially at $295 \mathrm{~K}$ and for pressures of 2 and 6 bar. The alignment factor, i.e. the average $<\cos (\theta)^{2}>(t)-1 / 3$ where $\theta$ is the angle between the molecular axis of symmetry (i.e. the $\mathrm{C}-\mathrm{C}$ axis) and the polarization of the laser, was computed since it is the quantity measured in Ref. 30. This was done with a time step of 1.5 fs for delays $\Delta t$ with respect to the pulse ranging from -2 ps to $90 \mathrm{ps}$. Note that the molecules were treated as rigid rotors in the ground vibrational state, thus disregarding the influence of centrifugal distortion and internal torsion and the contribution of the first excited state $\left(v_{4}\right)$. These approximations make things much simpler and they likely have negligible consequences on the dissipation of the alignment. Indeed, the latter results 
from rotational-state changes governed by state-to-state rates that are almost insensitive to slight changes of the energies and wave functions of the levels.

\section{B. Input data used}

We used the equilibrium values of the $\mathrm{C}_{2} \mathrm{H}_{6}$ geometry parameters, i.e. $\mathrm{C}-\mathrm{C}=1.535 \mathrm{~A}, \mathrm{C}$ $\mathrm{H}=1.094 \mathrm{~A}$ and $\mathrm{HCC}=111.2^{\circ}$, and the anisotropy of the polarizability was set to $\gamma=0.7 \mathrm{~A}^{3}$ as in Ref. 30. For the description of intermolecular forces, two potential energy surfaces were used, denoted as PES1 and PES2 and taken from Refs. 32 and 33, respectively. They were both computed $a b$ initio and represented by sums of site-site interactions described by analytical expressions. For the laser alignment pulse, we used the parameters reported in the experimental study ${ }^{30}$ : a duration (FWHM) of $100 \mathrm{fs}$ and a peak intensity of $80 \mathrm{TW} / \mathrm{cm}^{2}$. For comparisons with experiments, the computed alignment factor versus time was convoluted with a Gaussian function which takes into account the finite duration (150 fs) of the probe pulse and the crossing angle between the two beams.

\section{Requantization procedure}

While purely classical calculations can predict the response of the system during the laser pulse and the permanent alignment that it induces, they do not generate any alignment revivals since the latter are due to the quantum nature of the rotational motion (see Fig. 1a). A way to correct for this is to "requantize" the rotation in the course of the CMDS, following what was proposed 28 for $\mathrm{CO}_{2}$ and successfully tested against quantum calculations ${ }^{28}$ as well as experiments 29 . Before explaining how this was made in the case of the symmetric-top molecule ethane, recall that, under collision free conditions (zero pressure), the alignment factor computed quantum mechanically for a rigid rotor has a periodicity (see Fig. 1a of Ref. 30) equal to the rotational period $T_{r e v}=(2 B c)^{-1}=25.1 \mathrm{ps}$, where $\mathrm{B}=0.663 \mathrm{~cm}^{-1}$. Note that the weak revivals which appear ${ }^{30}$ at times $\Delta t=(n+1 / 4) T_{\text {rev }}$, with $\mathrm{n}=0,1,2$, will be disregarded from now on. They result from the different statistical weights of even and odd $J$ levels for $K=0$, and are not taken into account in our calculations. Within this limitation, all alignment revivals of odd number that appear at delays after the pulse of $\Delta t=(n+1 / 2) T_{\text {rev }}$, where $n=0,1,2, \ldots$, are identical. The same rule applies to those of even number occurring at $\Delta t=n T_{\text {rev }}$. Regarding the requantization of CMDS in the case of a symmetric-top molecule, recall that the rotational angular momentum in the molecular frame is 
$I_{x x} \omega_{x} \vec{x}+I_{y y} \omega_{y} \vec{y}+I_{z z} \omega_{z} \vec{z}$, with $I_{x x}=I_{y y} \neq I_{z z}, \vec{z}$ being the symmetry axis (along the C-C bond in $\mathrm{C}_{2} \mathrm{H}_{6}$ ). The requantization of the rotational motion around this axis, i.e. that of $\omega_{z}$, is straightforwardly obtained by introducing the rotational quantum number $K$ and remembering that, in the quantum world, one should have $\omega_{z}=\hbar K / I_{z z}$. Thus, at some specific times (see below) in the course of the CMDS we find the integer $K$ such that $\hbar K / I_{z z}$ is the closest to $\omega_{z}$ and then change $\omega_{z}$ to $\omega_{z}^{\mathrm{rq}}$ with $\omega_{z}^{\mathrm{rq}}=\left(\hbar K / I_{z z}\right)\left(\omega_{z} /\left|\omega_{z}\right|\right)$. For the components associated with the rotations around the $\mathrm{x}$ and $\mathrm{y}$ axes, and the requantization of $\omega_{x}$ and of $\omega_{y}$, we first wrote that, in the quantum world, one should have $\left(I_{x x} \omega_{x}\right)^{2}+\left(I_{y y} \omega_{y}\right)^{2}+\left(I_{z z} \omega_{z}\right)^{2}=\hbar^{2} J(J+1)$. Replacing $\omega_{z}$ by its requantized value $\omega_{z}^{\mathrm{rq}}$ into this equation and introducing $I_{\perp}=I_{x x}=I_{y y}$ leads to $I_{\perp}^{2}\left(\omega_{x}^{2}+\omega_{y}^{2}\right)=\hbar^{2}\left[J(J+1)-K^{2}\right]$. In order to requantize $\omega_{x}$ and $\omega_{y}$ we thus search for the integer $J$ such that $J(J+1)$ is the closest to $K^{2}+I_{\perp}^{2}\left(\omega_{x}^{2}+\omega_{y}^{2}\right) / \hbar^{2}$. We then replace $\omega_{x}$ by $\omega_{x}^{\mathrm{rq}}$ with $\omega_{x}^{\mathrm{rq}}=\left(\hbar / I_{\perp}\right) \sqrt{J(J+1)-K^{2}}\left(\omega_{x} / \sqrt{\omega_{x}^{2}+\omega_{y}^{2}}\right)$, an equivalent change being made for $\omega_{y}$. This requantization scheme, denoted as RQ1, leads to a practically perfect agreement with the results of quantum calculations (carried as explained in Ref. 30) for the first revival (and all other ones of odd number), as shown by Fig. 1b, but Fig. 1c shows that it does not reproduce the shape of the second revival (and of all those of even number). We then introduced a second requantization scheme, denoted as RQ2, in which we search for the integer $J$ such that $J^{2}$ is the closest to $K^{2}+I_{\perp}^{2}\left(\omega_{x}^{2}+\omega_{y}^{2}\right) / \hbar^{2}$ and then replace $\omega_{x}$ by $\omega_{x}^{\mathrm{rq}}$ with $\omega_{x}^{\mathrm{rq}}=\left(\hbar / I_{\perp}\right) \sqrt{J^{2}-K^{2}}\left(\omega_{x} / \sqrt{\omega_{x}^{2}+\omega_{y}^{2}}\right)$, an equivalent change being made for $\omega_{y}$. Figures $1 \mathrm{~b}$ and 1c show that, while RQ1 leads to very good (resp. inaccurate) results for revivals of odd (resp. even) numbers, the situation is reversed when RQ2 is used. 


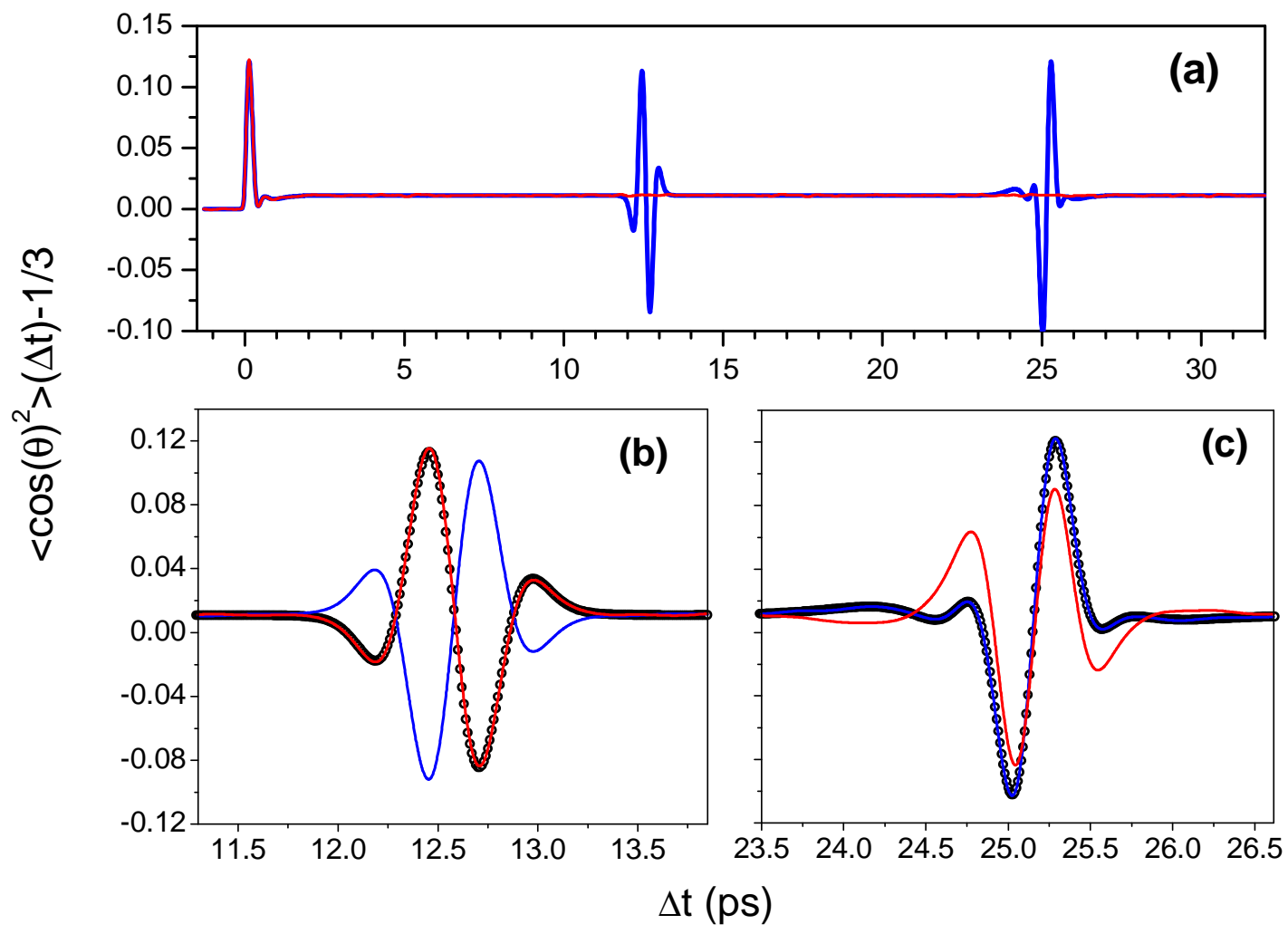

Fig. 1: Alignment factors for ethane gas under collision free conditions versus the time delay with respect to the laser pulse. The top panel (a) shows the results of a quantum calculation for rigid rotors in the ground vibrational state (blue line) and of fully classical MDS (red line). The lower panels display the same quantum results (circles) and those of requantized $C M D S$ using requantization $R Q 1$ (red line) and $R Q 2$ (blue line) for the first (b) and second (c) revivals.

In order to understand the results in Figs. $1 \mathrm{~b}$ and 1c, first note that one can show that, with RQ1, the ethane $\mathrm{C}-\mathrm{C}$ axis in the laboratory frame rotates with and angular speed $\omega_{\mathrm{RQ} 1}(J)=\hbar \sqrt{J(J+1)} / I_{\perp} \approx \hbar(J+1 / 2) / I_{\perp}$, while $\mathrm{RQ} 2$ leads to $\omega_{\mathrm{RQ} 2}(J)=\hbar J / I_{\perp}$. Obviously, if we consider the evolution of the square of a cosine between the molecular axis and the laser polarization direction, associated angular speeds are $2 \omega_{\mathrm{RQ} 1}(J) \approx \hbar(2 J+1) / I_{\perp}$ and $2 \omega_{\mathrm{RQ} 2}(J)=2 \hbar J / I_{\perp}$. Let us now consider the Raman transitions $J \rightarrow J+\Delta J$, with $\Delta J= \pm 1, \pm 2$, that are induced by the laser field and are responsible for the creation of the alignment revivals. They induce the oscillating terms $\exp \left(i \omega_{J, \Delta J} t\right)$ that appear in Eq. (15) of Ref. 30. Since $\omega_{J, J+\Delta J}=2 \pi B c[2 J \Delta J+\Delta J(\Delta J+1)]=\left(\hbar / 2 I_{\perp}\right)[2 J \Delta J+\Delta J(\Delta J+1)] \mid$, this leads, for the $\Delta J= \pm 1$ and $\Delta J= \pm 2$ to $\omega_{J, J \pm 1}=\hbar[J+(1 \pm 1) / 2] / I_{\perp}$ and 
$\omega_{J, J \pm 2}=\hbar(2 J+1 \pm 2) / I_{\perp}, \quad$ respectively. Hence, $\quad \omega_{J, J+2}=2 \omega_{\mathrm{RQ} 1}(J+1) \quad$ and $\omega_{J, J-2}=2 \omega_{\mathrm{RQ} 1}(J-1)$. This explains the good agreement between the quantum results and those obtained from rCMDS+RQ1 for the revivals of odd number (Fig. 1b) since only the $\Delta J= \pm 2$ Raman transitions contribute to the latter. Indeed, a re-alignment of a molecule at a delay $T_{r e v} / 2$ [or $(n+1 / 2) T_{r e v}$ with $n$ any integer value] after the pulse implies that the molecule rotates with an angular speed that is an even multiple of that corresponding to $T_{\mathrm{rev}}$ (i.e. to $J=1$ ) thus requiring that its quantum number $J$ is even. For the revivals of even number, all the $\Delta J= \pm 1$ and $\Delta J= \pm 2$ transitions contribute and the situation is much more complicated. While numerical computations show that rCMDS+RQ2 lead to good agreement with quantum calculations for the revivals of even number (see Fig. 1c), we have not been able to understand this result from analytical developments. It is important to emphasize that the quality of the predictions obtained with the two requantization procedures is largely due to the fact that many rotational levels (up to $J \approx 50$ ) are populated at room temperature. This "washes out", through averaging, the imperfections of RQ1 and RQ2. For the same reason, the total (rotational+translational) energy of the system remains remarkably constant after the laser pulse, despite the fact that the requantization changes the rotational speed of the molecules. Such a favorable situation would not occur if only few levels were populated, as it would be the case at (very) low temperature or for a molecule with a much larger rotational constant $B$. Indeed, comparisons similar to those made in Figs. 1b and 1c show a good agreement between the results of rCMDs and of quantum calculations down to about $10 \mathrm{~K}$ with differences that increase quickly for temperatures below $T<5 \mathrm{~K}$ as $T / B$ approaches unity.

It is not possible to find a single way to change the angular speeds of classically rotating molecules (i.e. to requantize) that can lead to accurate predictions of the revivals of both odd and even number. Hence, following the previously presented elements, we carried two requantized CMDS of $<\cos (\theta)^{2}>(t)-1 / 3$, using RQ1 and RQ2 respectively, for each given initial gas conditions and laser pulse characteristics. The final alignment factor was then obtained from a mix of the results obtained with the two requantizations. More precisely, we retained the alignment factor computed with RQ1 until $\Delta t=3 T_{\text {rev }} / 4$ and then in all the intervals $\left[(n+1 / 4) T_{\text {rev }},(n+3 / 4) T_{\text {rev }}\right]$ where $n=1,2,3, \ldots$ while that obtained with RQ2 was used in the intervals $\left[(n-1 / 4) T_{r e v},(n+1 / 4) T_{r e v}\right]$. Alternately using RQ1 and RQ2 is obviously not fully satisfactory, but this "trick" has the merit, as shown in Sec. III, to enable quantitative predictions of the collisional decays of the permanent and transient components 
of the alignment, without introducing any parameter adjustment,. Finally, the requantization (i.e., finding the values of $K$ and $J$ and then changing the rotational angular speeds $\left.\omega_{x}, \omega_{y}, \omega_{z}\right)$ is only made, for a given molecule, at times for which the torque that it is experiencing (due to the laser and/or interaction with another molecule) is practically zero. Indeed, requantizing at each time step would automatically forbid any change of the values of $J$ and $K$ and thus of the rotation of the molecule.

\section{RESULTS AND COMPRISONS WITH MEASUREMENTS}

A comparison between the measured alignment factor for pure ethane gas at 2 bar and initially at $295 \mathrm{~K}$ and that computed with the rCMDS approach and the intermolecular potential PES233 is shown in Fig. 2. As can be seen, the agreement between experiment and theory is good for the permanent alignment and the first two revivals but degrades for the higher order revivals. While it seems like the computations overestimate the decrease of the revivals amplitude with time, it is not truly the case. In fact, this does not come from an inaccurate modeling of the effect of collisions, as shown by the results for the decay time constants discussed below, but from the neglect of centrifugal distortion and of the contribution of the excited torsional state. Indeed, the comparison between quantum calculations displayed in Fig. 1 of Ref. 30 show that these contributions neglected in the rCMDS significantly reduce the amplitude of the $3^{\text {rd }}$ and $4^{\text {th }}$ revivals while broadening their extent but not those of the $1^{\text {rst }}$ and $2^{\text {nd }}$ one. The comparison of Figs. 1a and 1c of Ref. 30 also shows that these effects increase significantly as the revival number increases. 


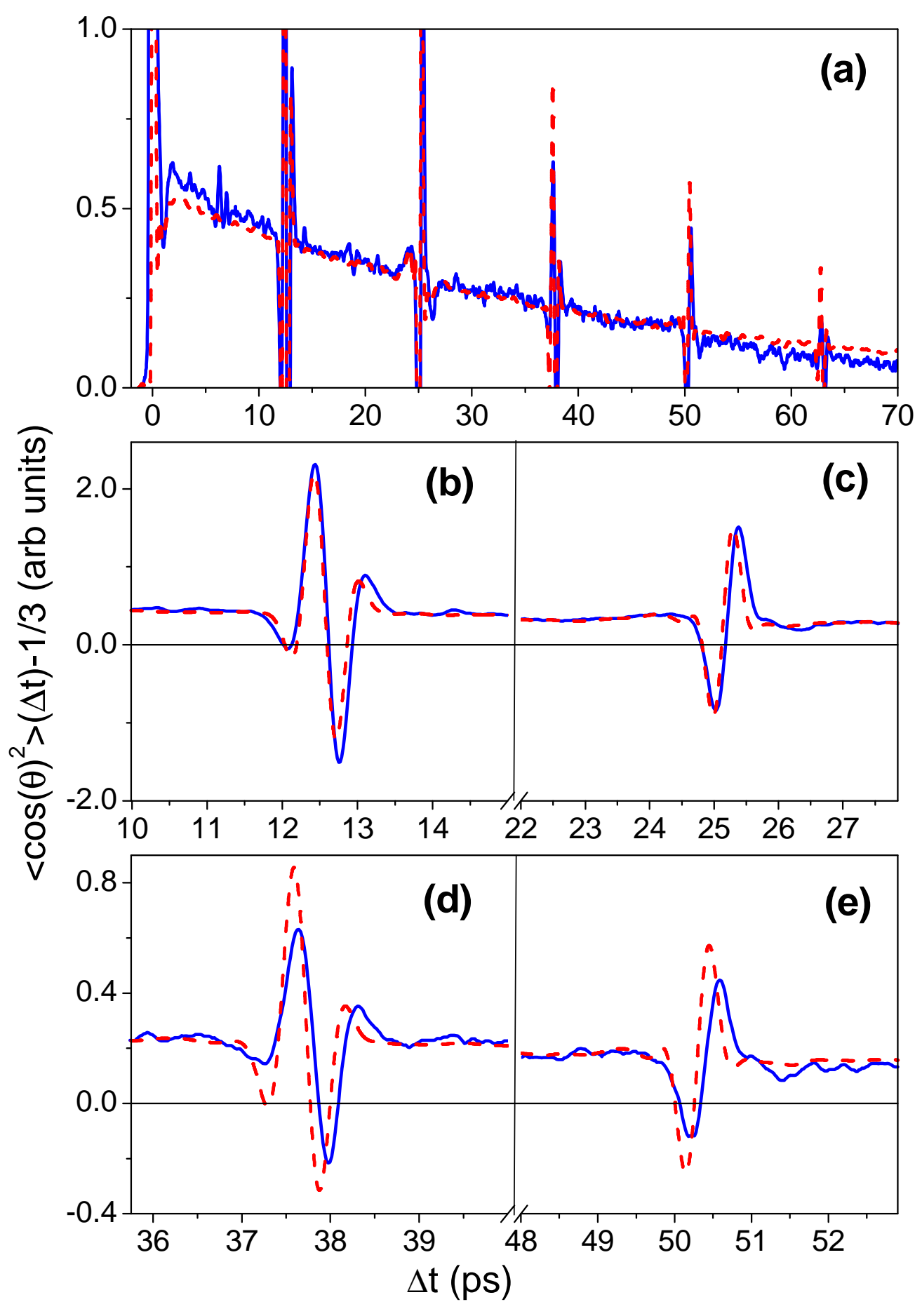

Fig. 2: Comparison between the measured (full blue line) alignment factor at 2 bar and that predicted by the requantized classical model (dashed red line) with the intermolecular potential PES233. The top panel (a) shows the results over the full time delay investigated, while the lower ones show detailed view around the first $(b)$, second $(c)$, third $(d)$, and fourth (e) revivals. 
In order to go further, we have determined the decay time constants of the permanent $\left(\tau_{\mathrm{P}}\right)$ and transient $\left(\tau_{\mathrm{T}}\right)$ alignments exactly as was done in Ref. 30. Namely, the permanent alignment was fit by an exponentially decaying function, yielding the value of $\tau_{\mathrm{P}}$. This function was then subtracted from the rCMDS-computed alignment factor and the absolute value of the result was calculated. We then determined the areas below each revival and fit them by another exponentially decaying function yielding the value of $\tau_{\mathrm{T}}$. The results of this exercise applied to rCMDS results at 2 bar are plotted in Fig. 3 which shows the quality of the exponential fits. A similar treatment was made for rCMDS results at 6 bar and, as expected from the binary nature of intermolecular collisions at such pressures, the time constants obtained are three times smaller than those given in the caption of Fig. 3. The values of $\tau_{\mathrm{P}}$ and $\tau_{\mathrm{T}}$ retrieved from the results of rCMDS carried using PES1 ${ }^{32}$ and PES2 ${ }^{33}$ have thus been density-normalized. They are given in Table 1 together with those obtained 30 from experiments. As can be seen the agreement between theory and measurements is very good when the intermolecular potential PES2 $2^{33}$ is used, The predicted values of $\tau_{\mathrm{T}}$ and $\tau_{\mathrm{P}}$ then differ from experimentally determined ones by only $-5.2 \%$ and $+7.7 \%$ and are within the uncertainties of the measurements. Poorer results are obtained with PES1 ${ }^{32}$. This could be expected since, with respect to PES1, the very recent PES2 is more accurate for several reasons, including the use of a much larger basis set, the fact that complete basis set extrapolations were made, that zero-point vibrational effects were not neglected, etc. As noted in Ref. 30, and confirmed by the present theoretical results, ethane follows the rule, previously evidenced for linear molecules $16,17,27,29$, that the permanent alignment decays more slowly than do the transient revivals. This was explained, for $\mathrm{CO}_{2}$, by a "gyroscopic effect" 28 resulting from the fact that collisions, while they change the rotational speed (i.e. the value of $J$ ), tend to conserve the direction of the rotational angular momentum (i.e. the value of $M / J$ ). A preliminary analysis, whose results will be presented in a forthcoming paper, shows that, for ethane, the situation is the same since the collisional cross sections for the $(J, K, M) \rightarrow\left(J^{\prime}, K^{\prime}, M^{\prime}\right)$ change have a maximum for $\mathrm{M}^{\prime} / \mathrm{J}^{\prime} \approx \mathrm{M} / \mathrm{J}$. 

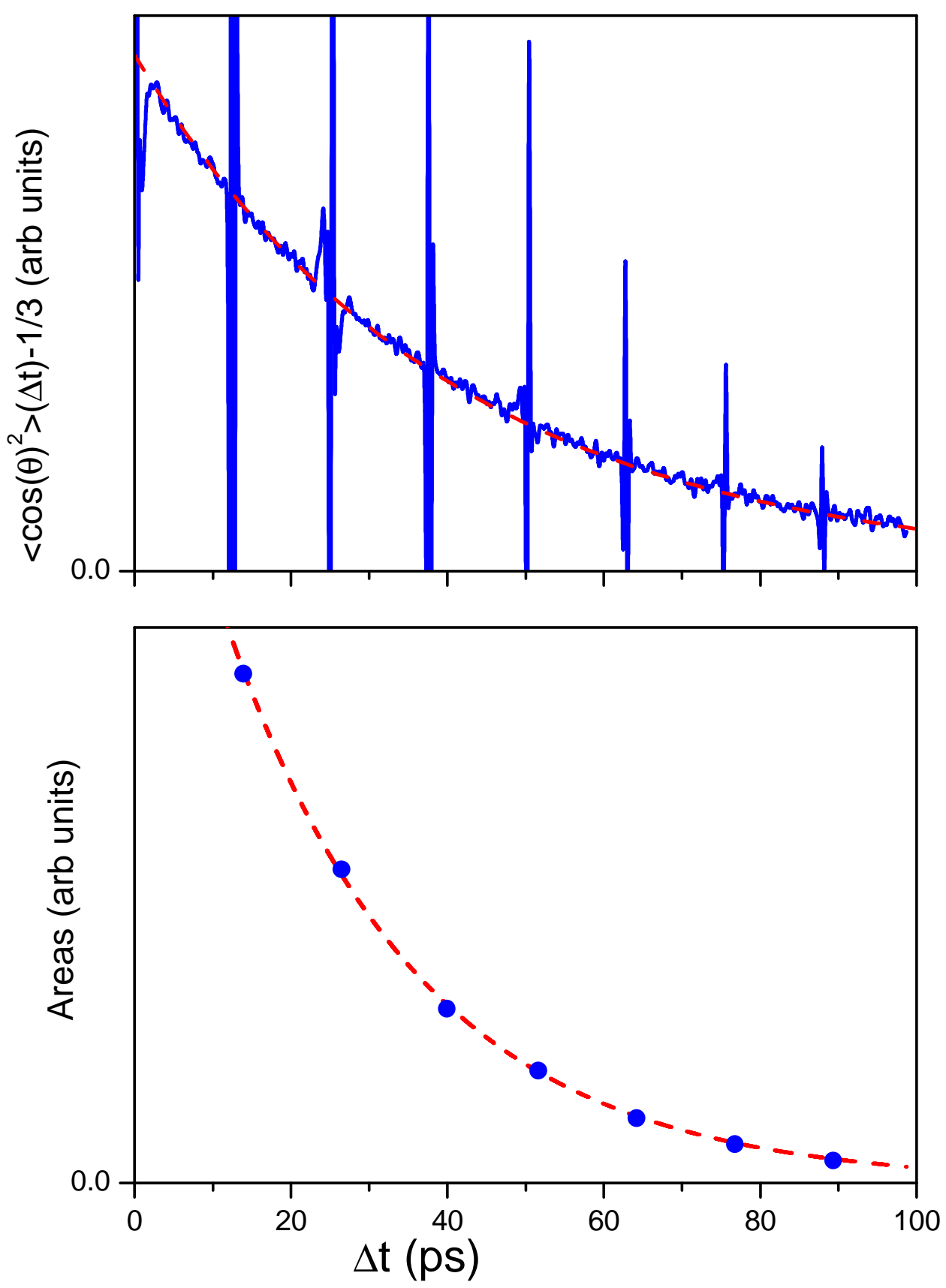

Fig. 3: Alignment factor (blue curve in top panel) and areas (see text) below the revivals (blue circles in bottom panel) obtained from rCMDS at 2 bar. The red dashed lines represent the associated exponential fits with time constants of $40.0 \mathrm{ps}$ and $24.7 \mathrm{ps}$, respectively. 


\begin{tabular}{|l|c|c|}
\hline & $\begin{array}{c}\tau_{\mathrm{P}} \\
(\text { ps. amagat })\end{array}$ & $\begin{array}{c}\tau_{\mathrm{T}} \\
\text { (ps. amagat) }\end{array}$ \\
\hline Exp $^{30}$ & $67.6 \pm 6.4$ & $47.4 \pm 3.6$ \\
\hline rCMDS (PES1 $\left.^{32}\right)$ & 91.0 & 47.9 \\
\hline rCMDS (PES2 & & \\
\hline
\end{tabular}

Table 1: Density-normalized time constants of the collision-induced decay of the permanent $\left(\tau_{\mathrm{P}}\right)$ and transient $\left(\tau_{\mathrm{T}}\right)$ alignment factor of ethane gas.

Finally, it is of interest to study how the symmetric-top nature of the ethane molecule affects the alignment and its collisional dissipation with respect to the behavior of linear species. In other words, what is the influence of the extra degree of freedom of rotation (that around the molecular axis of symmetry) brought by the fact that the molecule has a non zero moment of inertia $I_{\mathrm{zZ}}$. In order to investigate this issue, rCMDS have been carried using the true value of $I_{\mathrm{zz}}$ for ethane as well as with five times less and five times more. The results show that the collisional dissipation and the associated time constants $\tau_{\mathrm{P}}$ and $\tau_{\mathrm{P}}$ are practically insensitive to $I_{\mathrm{ZZ}}$. This can be explained by first noting that it is the component of the torque applied to a molecule in a collision with a partner that is perpendicular to the molecule symmetry $(\mathrm{C}-\mathrm{C})$ axis which changes the rotation of this axis and thus the alignment factor. Since $I_{\mathrm{xx}}=I_{\mathrm{yy}}$ for symmetric-tops, $I_{\mathrm{zz}}$ does not directly participate to changes of the associated speed of rotation [i.e. of $\left(\omega_{x}^{2}+\omega_{y}^{2}\right)^{1 / 2}$ ], as shown by Eqs. (3.29a,b) of Ref. 31. The effect of $I_{\mathrm{ZZ}}$ is thus only indirect, through its influence on the rotation around the C-C axis, which determines the relative positions of the $\mathrm{H}$ atoms of a colliding pair and thus the intermolecular forces. Now, it turns out that the potential energy surface of Ref. 33 is only weakly sensitive to the rotation around the $\mathrm{C}-\mathrm{C}$ axes of the two molecules. Its anisotropy is essentially governed by the respective orientation of the two $\mathrm{C}-\mathrm{C}$ axes so that this PES is more like that for two linear molecules. This explains the small sensitivity of the relaxation processes to the asymmetric-top nature of the considered molecule. This finding likely cannot be generalized to all symmetric-tops.

\section{CONCLUSION}

This paper presents the first model for calculations of the collisional dissipation of the laser-induced alignment of a gas of symmetric-top molecules. It is based on Classical 
Molecular Dynamics Simulations which are requantized through proper changes of the molecules' rotational speeds. It was applied to ethane gas for which measurements of the alignment have been made using a recent and accurate intermolecular potential energy surface. The comparison between predicted and measured alignment signals demonstrates the quality of the model. Indeed, the predicted decay rates of the permanent and transient alignments differ from experimentally determined ones by only $-5.2 \%$ and $+7.7 \%$ and are within error bars. The study of the influence of the symmetric-top geometry reveals that pure ethane gas behaves, from the point of view of the alignment dissipation, like a linear molecule. This results from the small sensitivity of the $\mathrm{C}_{2} \mathrm{H}_{6}-\mathrm{C}_{2} \mathrm{H}_{6}$ potential to rotations around the C-C axes, and is thus not a general rule for all symmetric-tops. It would thus be of interest to study, both experimentally and theoretically, the alignment of a gas species (pure or diluted gas) for which the potential sensitivity to rotations of the colliding molecules around their symmetry axes is large. However, finding a "good candidate" is not easy, very time consuming and my be unsuccessful because one needs to: (i) Find a symmetric-top + collision partner system for which a reliable intermolecular potential is available. (ii) This potential should be significantly anisotropic with respect to rotation around the molecule symmetry axis. (iii) The anisotropy of the polarizability of the symmetric-top molecule should be sufficient to enable a significant degree of alignment using current and reasonable laser pulse intensities. Although of interest, this search is beyond the scope of the present paper.

Acknowledgements: This work was supported by the Conseil Régional de Bourgogne under the PhotCom PARI program, the European Union (FEDER program), the Labex ACTION program (No. ANR-11-LABX-01-01), and the CoConiCS program (No. ANR-13-BS080013). 


\section{References}

1 H. Stapelfeldt, and T. Seideman, Rev. Mod. Phys. 75, 543 (2003).

2 M. Lemeshko, R. V. Krems, J. M. Doyle, and S. Kais, Mol. Phys. 111 (2013) 1648

3 T. Seideman, and E. Hamilton, Adv. At., Mol., Opt. Phys. 52, 289 (2005).

4 S. Fleischer, Y. Khodorkovsky, E. Gershnabel, Y. Prior, and I. S. Averbukh, Isr. J. Chem. 52, 414 (2012).

5 I. V. Litvinyuk, K. F. Lee, P. W. Dooley, D. M. Rayner, D. M. Villeneuve, and P. B. Corkum, Phys. Rev. Lett. 90, 233003 (2003).

6 J. Yao, G. Li, X. Jia, X. Hao, B. Zeng, C. Jing, W. Chu, J. Ni, H. Zhang, H. Xie, C. Zhang, Z. Zhao, J. Chen, X. Liu, Y. Cheng, and Z. Xu, Phys. Rev. Lett. 111 , 133001 (2013).

7 J. Itatani, J. Levesque, D. Zeidler, H. Niikura, H. Pepin, J. C. Kieffer, P. B. Corkum, and D. M. Villeneuve, Nature 432, 867 (2004).

8 X. Zhou, R. Lock, N. Wagner, W. Li, H. C. Kapteyn, and M. M. Murnane, Phys. Rev. Lett. 102, 073902 (2009).

9 O. Smirnova, Y. Mairesse, S. Patchkovskii, N. Dudovich, D. Villeneuve, P. Corkum, and M. Y. Ivanov, Nature 460, 972 (2009).

10 B. Shepperson, A. A. Søndergaard, L. Christiansen, J. Kaczmarczyk, R. E. Zillich, M. Lemeshko, and H. Stapelfeldt, Phys. Rev. Lett. 118, 203203 (2017).

11 P. W. Dooley, I. V. Litvinyuk, K. F. Lee, D. M. Rayner, M. Spanner, D. M. Villeneuve, and P. B. Corkum, Phys. Rev. A 68, 023406 (2003).

12 F. Rosca-Pruna and M. J. J. Vrakking, Phys. Rev. Lett. 87, 153902 (2001).

13 V. Renard, M. Renard, S. Guerin, Y. T. Pashayan, B. Lavorel, O. Faucher, and H. R. Jauslin, Phys. Rev. Lett. 90, 153601 (2003).

14 V. Renard, O. Faucher, and B. Lavorel, Opt. Lett. 30, 70 (2005).

15 T. Vieillard, F. Chaussard, D. Sugny, B. Lavorel, and O. Faucher, J. Raman Spectrosc. 39, 694 (2008).

16 T. Vieillard, F. Chaussard, F. Billard, D. Sugny, O. Faucher, S. Ivanov, J. M. Hartmann, C. Boulet, and B. Lavorel, Phys. Rev. A 87, 023409 (2013).

17 F. Chaussard, T. Vieillard, F. Billard, O. Faucher, J. M. Hartmann, C. Boulet, and B. Lavorel, J. Raman Spectrosc. 46, 691 (2015).

18 D. Pentlehner, J. H. Nielsen, A. Slenczka, K. Molmer, and H. Stapelfeldt, Phys. Rev. Lett. 110, 093002 (2013). 
19 J. Houzet, J. Gateau, E. Hertz, F. Billard, B. Lavorel, J. M. Hartmann, C. Boulet, and O. Faucher, Phys. Rev. A 86, 033419 (2012).

20 G. Karras, E. Hertz, F. Billard, B. Lavorel, J. M. Hartmann, and O. Faucher, Phys. Rev. A 89, 063411 (2014).

21 W. Schippers, E. Gershnabel, J. Burgmeier, O. Katz, U. Willer, I. S. Averbukh, Y. Silberberg, and W. Schade, Appl. Phys. B 105, 203 (2011).

22 B. Lavorel, P. Babilotte, G. Karras, F. Billard, E. Hertz, and O. Faucher, Phys. Rev. A 94, 043422 (2016).

23 H. Zhang, F. Billard, O. Faucher, and B. Lavorel, J. Raman Spectrosc. in press (2018)

24 S. Ramakrishna, and T. Seideman, Phys. Rev. Lett. 95, 113001 (2005).

25 S. Ramakrishna, and T. Seideman, J. Chem. Phys. 124, 034101 (2006).

26 N. Owschimikow, F. Konigsmann, J. Maurer, P. Giese, A. Ott, B. Schmidt, and N. Schwentner, J. Chem. Phys. 133, 044311 (2010).

27 S. Fleischer, Y. Zhou, R. W. Field, and K. A. Nelson, Phys. Rev. Lett. 107, 163603 (2011).

28 J. M. Hartmann, and C. Boulet, J. Chem. Phys. 136, 184302 (2012).

29 J. M. Hartmann, C. Boulet, T. Vieillard, F. Chaussard, F. Billard, O. Faucher, and B. Lavorel, J. Chem. Phys. 139, 024306 (2013).

30 H. Zhang, F. Billard, X. Yu, O. Faucher, and B. Lavorel, J. Chem. Phys. 148, 124303 (2018).

31 M.P. Allen, D.J. Tildesley. Computer simulation of liquids. Clarendon Press, Oxford, 1987.

32 R.L. Rowley, Yan Yang, and T.A. Pakkanen, J. Chem. Phys. 114, 6058 (2001)

33 R. Hellmann, J. Chem. Eng. Data 63, 470 (2018), 